THE JOURNAL OF INFECTIOUS DISEASES - VOL. 159, NO. 1 - JANUARY 1989

() 1989 by The University of Chicago. All rights reserved. 0022-1899/89/5901-0010\$01.00

\title{
AIDS COMMENTARY
}

\section{Tuberculosis and Human Immunodeficiency Virus Infection}

\author{
Richard E. Chaisson and Gary Slutkin
}

\begin{abstract}
From the Departments of Medicine and Epidemiology, Johns Hopkins University Schools of Medicine and Hygiene and Public Health, Baltimore, Maryland; and the Special Programme on AIDS, World Health Organization,
\end{abstract}

Geneva, Switzerland

\begin{abstract}
NOTE From DR. MERle A. SANDE - Progressive human immunodeficiency virus infection eventually leads to activation and dissemination of a wide variety of microorganisms normally held in check by the cellular immune system. Mycobacterium tuberculosis is one of these pathogens, and the disease caused by it has become a common presenting infection in the patient with AIDS. Dr. Richard E. Chaisson and Dr. Gary Slutkin have studied tuberculosis in the United States and worldwide, respectively. In this AIDS Commentary they address the unique nature of this infection, its diagnosis, and its treatment in the patient with AIDS.
\end{abstract}

Infection with human immunodeficiency virus (HIV) ultimately has a profound effect on cellmediated immunity and thus increases the risk of diseases caused by pathogens that are normally held in check by this defense mechanism. It is therefore logical to assume that persons with HIV infection are predisposed to tuberculosis, especially if they have been previously infected with Mycobacterium tuberculosis. Presently, there is no definitive substantiation of a causal relation between HIV infection and tuberculosis, but significant epidemiological information strongly suggests that this relation exists.

In the United States, since 1984 the rate of decline in tuberculosis cases has halted, and in fact, in 1986 the number of cases of tuberculosis increased by $2.6 \%$ from 22201 to 22768 [1]. The Division of Tuberculosis Control of the CDC estimates that in 1985-1987 there were $>9000$ excess cases of tuberculosis over the expected number of cases, had the rate of decline seen in 1980-1984 continued. The change in the tuberculosis case-rate is especially marked in areas where there is a high prevalence of HIV infection, such as New York City, Florida, and California. Among United States-born residents of San Francisco, for example, there was a doubling in the incidence of tuberculosis from 1982 to 1987 [2].

In 1985 the CDC modified the surveillance defi-

Please address requests for reprints to Dr. Richard E. Chaisson, Division of Infectious Diseases, Johns Hopkins Hospital, 1830 East Monument Street, Suite 7400, Baltimore, Maryland 21205. nition of AIDS to include extrapulmonary tuberculosis in any person known to be seropositive for HIV [3]. Tuberculosis at any site occurs in $\sim 2 \%$ of AIDS cases in San Francisco, 5\% of AIDS cases in New York City, and $10 \%$ of AIDS cases in Florida $[4,5]$. In addition, Mycobacterium kansasii, a nontuberculous mycobacterium that causes disease that is clinically indistinguishable from that caused by $M$. $t u$ berculosis, has been reported in a small number of patients with AIDS. Several recent studies indicate that the scope of tuberculosis as a complication of HIV infection may be far greater than previously appreciated. In studies of newly diagnosed cases of tuberculosis in Miami, Kinshasa (Zaire), and San Francisco, $\sim 30 \%$ of patients were found to be HIV seropositive, although most lacked other clinical signs of AIDS [6-8]. As more clinicians perform serological tests for HIV in patients with tuberculosis, it is likely that a large portion will be found to have both infections.

Which patients with HIV infection develop tuberculosis? There is no evidence that HIV infection predisposes patients to acquisition of $M$. tuberculosis infection. Persons with prior $M$. tuberculosis infection are, however, more likely to develop tuberculosis if they are coinfected with HIV. Selwyn and associates in New York found [9] that $15 \%$ of HIVseropositive iv drug abusers with a history of a positive tuberculin skin test developed tuberculosis over a two-year period, compared with none of a similar group of HIV-seronegative individuals. The populations of patients that can be expected to develop 
tuberculosis are those with a high prevalence of coinfection with HIV and $M$. tuberculosis. In developed countries this includes HIV-seropositive persons who are immigrants from countries with endemic tuberculosis, intravenous drug abusers, poor inner city residents (particularly members of ethnic minorities), and close contacts of individuals with tuberculosis. Screening of these populations with tuberculin skin tests is an urgent public health priority. In developing countries the overall prevalence of tuberculous infections (positive tuberculin tests) may exceed $50 \%$, a fact making it very likely that a substantial proportion of HIV-seropositive persons will have both infections.

What is the clinical presentation? Tuberculosis in HIV-infected patients, as in other populations, is primarily a pulmonary infection. Early reports of AIDS-related tuberculosis focused on the atypical clinical features found in patients with advanced HIV disease, particularly extrapulmonary sites of disease, skin test anergy, and diffuse infiltrates on chest radiographs [10-13]. Subsequent experience has shown that many HIV-associated cases of tuberculosis occur earlier in the course of immunosuppression and have features that are more characteristic of "standard" tuberculosis. When tuberculosis occurs before another opportunistic infection, $75 \%-100 \%$ of patients have pulmonary disease. Tuberculosis occurring after a diagnosis of AIDS has been made is still most often pulmonary, although extrapulmonary sites are becoming more commonly involved. Between $25 \%$ and $70 \%$ of cases of HIV-associated tuberculosis include an extrapulmonary site of disease, a finding that fulfills the CDC surveillance criteria for AIDS. It is likely that extrapulmonary tuberculosis portends a poorer outcome than does pulmonary tuberculosis alone, because extrapulmonary cases appear to have a greater degree of immunodeficiency as measured by $\mathrm{CD} 4^{+}$cell counts and the presence of oral candidiasis.

The symptoms of tuberculosis in HIV-seropositive patients are similar to those encountered in other patients. Fever and night sweats are the most common complaints, and other constitutional symptoms, such as weight loss, anorexia, and chills, are frequently reported. Cough, sputum production, and dyspnea are noted by most patients with pulmonary disease; pleuritic chest pain may also occur. Signs and symptoms specific to extrapulmonary sites of disease, such as localized lymphadenopathy or meningismus and headache, are usually experienced by patients found to have tuberculosis outside of the lungs. The duration of signs and symptoms may vary but is usually at least several weeks, and it may be difficult to distinguish the symptoms of tuberculosis from those of Pneumocystis carinii pneumonia or other opportunistic infections. The abrupt onset of pulmonary symptoms is unusual in tuberculosis and is more suggestive of pyogenic pneumonia. A chronic wasting syndrome without any localizing signs or symptoms may be the only clue to tuberculosis in any patient with or without HIV infection.

Physical examination is usually nonspecific and may reveal fever, tachycardia, tachypnea, oral thrush or hairy leukoplakia, and generalized or regional lymphadenopathy. Chest examination often shows localized consolidation, rales, and dullness, although no abnormalities may be discerned in a number of patients. Hepatosplenomegaly is frequently found in HIV-seropositive individuals and is nonspecific for tuberculosis. The diagnosis of tuberculosis in HIV-infected patients, as in all groups of patients, depends upon suspicion by the physician and inclusion of tuberculosis in the differential diagnosis of opportunistic diseases associated with HIV infection. Failure to perform appropriate diagnostic tests in HIV-seropositive patients who present with a pulmonary or systemic illness will result in underdiagnosis and undertreatment of tuberculosis.

How should patients be evaluated? Chest radiographs should be performed on all patients with HIV infection who present with respiratory or constitutional symptoms. The radiographic appearance of tuberculosis in HIV disease may vary with the degree of immunodeficiency. In patients with moreadvanced HIV disease (e.g., low $\mathrm{CD} 4^{+}$lymphocyte counts, other opportunistic diseases), the chest radiograph most often has diffuse pulmonary infiltrates $(\sim 60 \%)$, although focal consolidation may also be present $(\sim 30 \%-40 \%)$. Cavitary lesions are uncommon in these patients. Intrathoracic adenopathy may be found in up to $25 \%$ of patients with AIDS-related tuberculosis and should raise the index of suspicion, because neither $P$. carinii pneumonia nor persistent generalized lymphadenopathy is associated with mediastinal adenopathy. Pleural effusion is found in $<25 \%$ of patients. Several patients with AIDS who had documented pulmonary tuberculosis have been reported to have normal chest radiographs at presentation. In patients with less HIVinduced immunosuppression, chest radiographs are more "classic," with upper lobe infiltrates that may 
cavitate ( $\sim 45 \%)$; lower lung infiltrates without cavitation $(\sim 20 \%)$, mediastinal adenopathy $(\sim 20 \%$ $30 \%$ ), and pleural effusion $(<10 \%)$ are also seen in this population. The clinical evaluation of pulmonary abnormalities in patients with HIV infection has been recently reviewed [14]. Tuberculosis should be included in the differential diagnosis of all pulmonary disease processes and abnormalities in such patients, and mycobacterial stains and cultures should be routinely performed on all diagnostic specimens obtained during the evaluation. For example, specimens of induced sputum should be stained for acid-fast bacilli and cultured for mycobacteria in addition to being stained for $P$. carinii. Similarly, bronchoalveolar lavage and transbronchial biopsy specimens should also be stained and cultured for mycobacteria. Approximately $50 \%$ of patients with pulmonary tuberculosis that occurs earlier in HIV infection have positive acid-fast smears, whereas up to $75 \%$ of patients with more-advanced HIV disease will have positive smears. Because culture results may often be delayed, a positive acid-fast smear should be presumed to be $M$. tuberculosis, and therapy should be initiated while awaiting culture confirmation. Patients with a clinical presentation suggestive of tuberculosis who have negative acid-fast smears should be empirically treated for tuberculosis until culture results are available. Use of the BACTEC ${ }^{\circledR}$ culture system (Johnston Laboratories, Towson, Md) may considerably accelerate the recovery and identification of mycobacteria.

Tuberculin skin testing with 5 tuberculin units (TU) of PPD (purified protein derivative) should be performed in all HIV-infected patients with suspected tuberculosis. Although many patients with advanced HIV infection may be anergic, one-third to one-half of patients with AIDS and tuberculosis have a $>10-\mathrm{mm}$ reaction to PPD. Fifty percent to $80 \%$ of HIV-seropositive patients with tuberculosis but without AIDS react to PPD. In patients with HIV infection and suspected tuberculosis, a $>5-\mathrm{mm}$ reaction to PPD should be considered indicative of tuberculous infection. Serological tests, such as an enzyme immunoassay for antibodies to PPD, show some promise in diagnosing tuberculous infection in both immunocompetent and immunodeficient patients, but these tests are not routinely available.

Extrapulmonary tuberculosis may be present alone or in addition to pulmonary tuberculosis in patients with HIV infection. The most common sites involved are lymph nodes, blood and bone marrow, urinary tract, liver, and CNS. When clinical findings or laboratory studies suggest disease at an extrapulmonary site, aspiration or biopsy of the involved organ should be done and acid-fast smears and mycobacterial cultures performed. Fine-needle aspiration of lymph nodes is an acceptable alternative to biopsy when peripheral adenopathy is present. Mediastinal adenopathy can be approached by transbronchial needle aspiration or mediastinoscopy. Pleural effusion should be aspirated, and pleural fluid should be evaluated for cell count and chemistry values as well as stained and cultured for pyogenic bacteria and mycobacteria. A closed pleural biopsy may be necessary to diagnose tuberculous pleurisy. CNS disease can produce ring-enhancing lesions on computed tomographic scans; a biopsy should be performed if there is no response to empiric treatment of toxoplasmosis. The diagnosis of tuberculosis at an extrapulmonary site should prompt evaluation for pulmonary involvement by using chest radiographs and sputum induction for mycobacterial studies.

How should HIV-associated tuberculosis be treated? Standard antituberculosis drugs are extremely effective for treating tuberculosis in patients with HIV infection. Most $M$. tuberculosis isolates from patients with AIDS are susceptible to the firstline agents. The recommended regimen for treating tuberculosis in $\mathrm{HIV}$-infected adult patients is isoniazid $(300 \mathrm{mg} / \mathrm{d})$, rifampin $(600 \mathrm{mg} / \mathrm{d} ; 450 \mathrm{mg} / \mathrm{d}$ for persons weighing $<50 \mathrm{~kg}$ ), and pyrazinamide $(20-30$ $\mathrm{mg} / \mathrm{kg}$ per day). Ethambutol (15-25 mg/ $\mathrm{kg}$ per day) may also be used if isoniazid resistance is suspected. Pyrazinamide and ethambutol should be discontinued after two months of therapy if the isolate is isoniazid susceptible, whereas isoniazid and rifampin are given for a total of six to nine months. Isoniazid and rifampin alone given for nine to 12 months is also an acceptable regimen. The CDC and the American Thoracic Society recommend that treatment be given for at least six months beyond conversion of sputum cultures to negative in patients with HIV infection and pulmonary tuberculosis [15]. Patients not treated with isoniazid or rifampin should receive at least 18 mo of therapy. Extrapulmonary disease can also be treated with standard regimens, including isoniazid and rifampin, and therapy should be continued for at least nine to 12 months. There are anecdotal reports of meningeal or CNS infections that fail to respond or that prog- 
ress during antituberculosis therapy. Addition of second-line agents such as ethionomide or cycloserine may be necessary in such situations.

Clinical experience in treating HIV-associated tuberculosis with standard short-course chemotherapy suggests that the response is very favorable, usually resulting in rapid resolution of symptoms, clearing of radiographic abnormalities, and sterilization of sputum cultures at the same rate as in HIV-seronegative patients [8]. Nevertheless, the appropriate duration of antituberculosis therapy remains controversial, with some authorities arguing for lifelong treatment with isoniazid after the completion of acute therapy with multiple agents. The CDC and the American Thoracic Society currently recommend treatment for at least six months after conversion of cultures to negative [15]. Clinical trials addressing the value of lifelong treatment are being planned but may be difficult to perform because of the large sample sizes required and competing mortality from other AIDS-related illnesses. At the San Francisco Department of Health Tuberculosis Clinic, 71 patients with AIDS have been treated with short-course chemotherapy (six to nine months) without maintenance therapy; only two patients (one of whom was noncompliant) had a relapse after antituberculosis drug therapy was discontinued (G. F. Schecter, personal communication). Another patient had progressive disease with CNS dissemination while receiving therapy for an isoniazid-resistant infection.

Patients who are noncompliant or who fail to complete a full course of therapy are, however, at increased risk for relapsing, and supervised therapy should be given to individuals who are considered to be unreliable or who are unable to manage their medications because of CNS dysfunction or other impairment. Patients should have follow-up chest radiographs and sputum cultures at the completion of treatment and again six months later. Patients with advanced HIV disease may have more-adverse reactions to antituberculosis agents, although this is not the case for HIV-infected patients who have tuberculosis but do not have AIDS [4, 8].

Although tuberculosis in HIV-seropositive patients can be easily managed with appropriate antibiotics, other opportunistic infections may supervene during or after therapy. A patient who appears to be failing or relapsing may have $P$. carinii pneumonia or disease caused by another pathogen and should be thoroughly evaluated. The cause of death in patients with AIDS and tuberculosis is almost always another opportunistic infection rather than M. tuberculosis.

Should preventive therapy be given to PPDpositive patients with HIV infection? The risk of developing tuberculosis for a person with HIV infection is substantial. Consequently, chemoprophylaxis is recommended for any HIV-seropositive individual who has a significant reaction to $5 \mathrm{TU}$ of intradermal PPD [15]. Persons with a documented history of a positive tuberculin skin test should also receive preventive therapy. Tuberculin skin testing should be performed on HIV-infected patients when they are diagnosed as seropositive, particularly in patients from populations with an increased risk of prior $M$, tuberculosis infection. Although it is possible that some infected patients will be anergic, data from HIV-seropositive individuals with tuberculosis cited above suggest that many, if not most, patients will react to PPD. HIV serological testing should be considered for patients found to have a positive tuberculin test, particularly for individuals who might not otherwise receive preventive therapy (e.g., $>35$ y old). Patients with a positive skin test should have a chest radiograph to evaluate the presence of active disease; some clinicians would recommend sputum cultures in all patients regardless of the appearance of the chest radiograph, although the likelihood of a positive culture from an asymptomatic person is very low.

Isoniazid $(300 \mathrm{mg} / \mathrm{d})$ is the preferred agent for prophylaxis, except in individuals in close contact with a patient with disease that is caused by an isoniazidresistant organism. The optimal duration of preventive therapy is not known. Although isoniazid should be administered for at least six to 12 months, some clinicians would favor lifelong chemoprophylaxis in patients with HIV infection because of the inevitability of progressive deterioration of cellular immune function in this population. Currently, there are no reliable data upon which to base a decision, although we are not aware of reports of failure of preventive therapy given for six to 12 months.

Contact tracing is an essential component of tuberculosis control programs and is especially important for HIV-associated cases. Close contacts of cases should be evaluated with a tuberculin skin test. If an independent risk for HIV infection is present, HIV antibody testing should also be of fered. Contact tracing for HIV-seropositive patients with a sig- 
nificant reaction to PPD may also be useful in identifying untreated individuals with tuberculosis.

\section{References}

1. Centers for Disease Control. Tuberculosis, final data-United States, 1986. MMWR 1988; 36:817-20

2. Tuberculosis in San Francisco, 1987: final data. San Francisco Epidemiological Bulletin 1988;4:15-6

3. Centers for Disease Control. Revision of the CDC surveillance case definition for acquired immunodeficiency syndrome. MMWR 1987;36(Suppl):1S-15S

4. Chaisson RE, Schecter GF, Theuer CP, Rutherford GW, Echenberg DF, Hopewell PC. Tuberculosis in patients with acquired immunodeficiency syndrome: clinical features, response to therapy and survival. Am Rev Resp Dis 1987; 136:570-4

5. Centers for Disease Control. Tuberculosis and acquired immunodeficiency syndrome-New York City. MMWR 1987; 36:785-95

6. Pitchenik AE, Burr J, Suarez M, Fertel D, Gonzalez G, Moas C. Human T-cell lymphotrophic virus III (HTLV-III) seropositivity and related disease among 71 consecutive patients in whom tuberculosis was diagnosed. Am Rev Resp Dis 1987;135:875-9

7. Mann J, Snider DE, Francis H, Quinn TC, Colebunders RL, Piot P, Curran JW, Nzilambi N, Bosenge N, Malonga M, Kalunga D, Nzingg MM, Bagala N. Association between HTLV-III/LAV infection and tuberculosis in Zaire. JAMA 1986;256:346
8. Theuer CP, Chaisson RE, Schecter GF, Hopewell PC. Human immunodeficiency virus infection in tuberculosis patients in San Francisco. Am Rev Resp Dis 1988;137:121a

9. Selwyn PA, Lewis VA, Schoenbaum EE, Hartel D, Klein RS, Freidland GH. HIV infection and tuberculosis in intravenous drug users (IVDU) in a methadone program (MMTP) [abstract 7549]. In: Proceedings of the IVth International Conference on AIDS. Stockholm, Sweden, 1988

10. Pitchenik AE, Cole C, Russell BW, Fischl MA, Spira TJ, Snider DE. Tuberculosis, atypical mycobacteriosis, and the acquired immunodeficiency syndrome among Haitian and non-Haitian patients in South Florida. Ann Intern Med 1984; 101:641-5

11. Sundcram G, McDonald RJ, Maniatis T, Oleske J, Kapila $R$, Reichman LB. Tuberculosis as a manifestation of the acquired immunodeficiency syndrome (AIDS). JAMA 1986;256:362-6

12. Louie E, Rice LB, Holzman RS. Tuberculosis in non-Haitian patients with acquired immunodeficiency syndrome. Chest 1986;90:542-5

13. Handwerger S, Mildvan D, Senie R, McKinley FW. Tuberculosis and the acquired immunodeficiency syndrome at a New York City Hospital: 1978-1985. Chest 1987;91:176-80

14. Hopewell PC. Pneumocystis carinii pneumonia: diagnosis. J Infect Dis 1987;157:1115-9

15. Centers for Disease Control. Diagnosis and management of mycobacterial infection and disease in persons with human T-lymphotropic virus type III/lymphadenopathyassociated virus infection. MMWR 1986;35:448-52 